\title{
Uma coleção de artigos científicos de Português compondo um Corpus no domínio educacional
}

\author{
LUÍS HENRIQUE G. DE AGUIAR
}

Mestrando no Programa de Pós-graduação em Educação da Universidade Federal dos Vales do Jequitinhonha e Mucuri (UFVJM). E-mail: luishenriqueaguiar@gmail.

\section{VALDIR JR. CORDEIRO ROCHA}

Mestrando no Programa de Pós-graduação em Educação da Universidade Federal dos Vales do Jequitinhonha e Mucuri (UFVJM). E-mail: vjcordeiror@gmail.com

\section{MARCUS VINÍ́CIUS C. GUELPELI}

Professor Doutor da Universidade Federal dos Vales do Jequitinhonha e Mucuri (UFVJM).

E-mail: marcus.guelpeli@ufvjm.edu.br 
Resumo: Este artigo descreve a construção de um corpus de textos, o qual é formado por artigos científicos, no domínio educacional, trazendo as estatísticas que o compõem. Com este trabalho, pretendese obter um corpus que torne possíveis diversas pesquisas na área de Processamento de Linguagem Natural e especificamente na área de Sumarização Automática, para possibilitar análise da performance de sumarizadores na língua portuguesa.

Palavras-chave: Corpus. Linguística Computacional. PLN. Sumarização.

\begin{abstract}
This article describes the construction of a Portuguese texts corpus in the educational domain. The corpus consists of scientific articles. The work also brings the statistics that make up this corpus. This work aims to obtain a corpus which make possible several research in natural language processing area and specifically in Automatic Summarization area to enable analysis of summarizers performance in Portuguese.
\end{abstract}

Keywords: Corpus. Computational Linguistics. PLN; Summarization.

\title{
Introdução
}

O termo corpus é usado para fazer referência a uma coleção cujos textos são escritos, armazenados eletronicamente e processados por computador com propósitos de pesquisa linguística. Ao construir um corpus de textos, procura-se fazer uma seleção de dados representativa, isto é, que constitua um corpo de evidências linguísticas que possa suportar generalizações e contra as quais se possam testar hipóteses (MARTINS, CASELI e NUNES, 2001).

Segundo Zavaglia e Ferraresi (2006), a Linguística de Corpus (LC) exerce ampla influência na pesquisa linguística, principalmente na Europa. No Brasil, esse tipo de pesquisa ainda está em estágio inicial, mas não deixa de ocorrer, por exemplo, em centros especializados em Processamento de Linguagem Natural, Lexicografia e Linguística Computacional.

Este trabalho relata a construção de um corpus, que adota a língua portuguesa, de domínio educacional, formado por 10 subcategorias. Nas pesquisas, utilizaram-se repositórios acadêmicos e adotou-se um critério segundo o qual os arquivos deveriam possuir resumos e palavras-chave formados por seus autores, já que se pretende com este corpus realizar futuras pesquisas na área de Sumarização Automática (SA). 
Este trabalho está dividido em cinco seções. A seção dois define e classifica o termo corpus; na seção três, apresenta-se a metodologia aplicada na construção do corpus e a quarta seção expõe suas estatísticas.

\section{Corpus}

Segundo Rebechi e Andreetto (2015), a LC é uma disciplina que se ocupa da investigação de unidades convencionais da língua em um texto ou conjunto de textos em formato eletrônico, ou seja, daquilo que é mais provável de ocorrer na língua, não necessariamente daquilo que é apenas possível. Uma das vantagens da observação dos dados por meio de corpora é que ela revela evidências empíricas e sistemáticas da linguagem, as quais poderiam passar despercebidas sem o auxílio de ferramentas computacionais específicas para esse tipo de investigação. Além disso, uma pesquisa puramente intuitiva pode levar o pesquisador a "encontrar" dados que simplesmente "comprovem" suposições ou hipóteses previamente estabelecidas, sem revelar dados novos que possam levar a outras descobertas.

Segundo Oliveira e Guelpeli (2014), a palavra corpus (plural: corpora) tem origem no latim corpo, conjunto de textos, que em LC determina uma coleção de textos selecionados e organizados. Segundo Sardinha (2000), a Linguística de Corpus ocupa-se da coleta e exploração de corpora, ou conjunto de dados coletados de forma criteriosa com a finalidade de serem utilizados para pesquisa de uma língua ou variedade linguística.

Em 1961 foi construído o primeiro corpus linguístico eletrônico denominado Brown (KUCERA e FRANCIS, 1961), formado por 1 milhão de palavras, impulsionando o desenvolvimento da LC. Para Aluísio e Almeida (2006), um corpus computadorizado observa um conjunto de considerações que influencia a validade e confiabilidade da pesquisa baseada em corpus, sendo a sua criação um processo repetitivo. Tal processo começa com a seleção dos textos, baseada em algum critério significativo para a pesquisa (critério externo), continua com as investigações empíricas da língua ou variedade linguística sob análise (critério interno) e, por fim, tem-se a revisão de todo o projeto.

Para Humblé (2001), um corpus pode ser compreendido como uma quantidade grande de textos armazenados no computador e que são acessados com programas próprios de pesquisa. Esses dados podem ser variados, como jornais, bulas de remédios ou artigos científicos, sendo 
obtidos de diversas fontes, dependendo do contexto e necessidade, sendo os mais comuns os textos digitalizados e retirados da internet.

\section{Metodologia}

Esta seção descreve a metodologia de construção do que foi proposto, adotando a descrita por Aluísio e Almeida (2006), que divide em três estágios a compilação de um corpus próprio. Primeiramente é a fase de projeto, que inclui a seleção dos textos, logo após realiza-se a captura, manipulação, nomeação dos arquivos e, por fim, efetua a anotação. A manipulação dos textos, que abrange a limpeza e formatação do corpus para o processamento computacional, bem como a organização estrutural deste, segue a metodologia proposta por Guelpeli (2012).

A compilação do corpus teve duração de quatro meses (dezembro de 2015 a março de 2016) e para tanto foi utilizada a tabela de áreas de conhecimento da grande área da Educação, para a definição de dez subáreas que compõem as categorias do corpus. A referida tabela encontra-se disponível no site da Coordenação de Aperfeiçoamento de Pessoal de Nível Superior (CAPES) 1. As categorias escolhidas foram: Educação Especial; Educação Permanente; Educação Préescolar; Ensino-aprendizagem; Filosofia da Educação; História da Educação; Política Educacional; Psicologia Educacional; Sociologia da Educação e Tecnologia Educacional.

$\mathrm{Na}$ fase de projeto, denominada por Aluísio e Almeida (2006) critério externo, ocorreu a seleção dos textos, observando os critérios de (1) gratuidade; (2) possibilidade de reprodução dos arquivos originais; (3) classificação das bases para o domínio e subáreas escolhidas para a pesquisa; (4) resumo do texto original, denominado sumário de referência, elaborado pelo autor. Foram escolhidos somente artigos científicos, por possuírem resumo e palavras-chave, que poderão ser utilizados posteriormente para testes com sumarizadores automáticos de texto. Os artigos científicos foram retirados, em sua maioria, do repositório Scientific Electronic Libary online (SCIELO) ${ }^{2}$, nas categorias já mencionadas. As categorias em que não houve preenchimento total pelos artigos do repositório da SCIELO - Educação permanente; Ensino aprendizagem; Filosofia da Educação; Política Educacional e Sociologia da Educação - foram preenchidas por

1Disponível em: http://www.capes.gov.br/avaliacao/instrumentos-de-apoio/tabela-de-areas-do-conhecimento-avaliacao

2 Disponível em: http://www.scielo.br/ 
artigos encontrados no repositório Buscador Coruja ${ }^{3}$, além disso, o domínio Educação Pré-escolar também foi preenchido com artigos encontrados segundo pesquisa com termos relevantes feitas no Google.

Na segunda fase, conforme realizado por Guelpeli (2012), ocorreram a limpeza e a formatação do corpus para o processamento computacional. Foi retirado tudo que não fazia parte do texto - gráficos, tabelas, figuras e números de páginas. Os arquivos fontes foram convertidos do formato em "PDF" para o "TXT", que é compatível para o processamento. Para cada uma das 10 categorias que compõem o domínio, criou-se uma subpasta, conforme exemplifica a Figura 1.

Figura 1. Categorias que compõem o domínio.

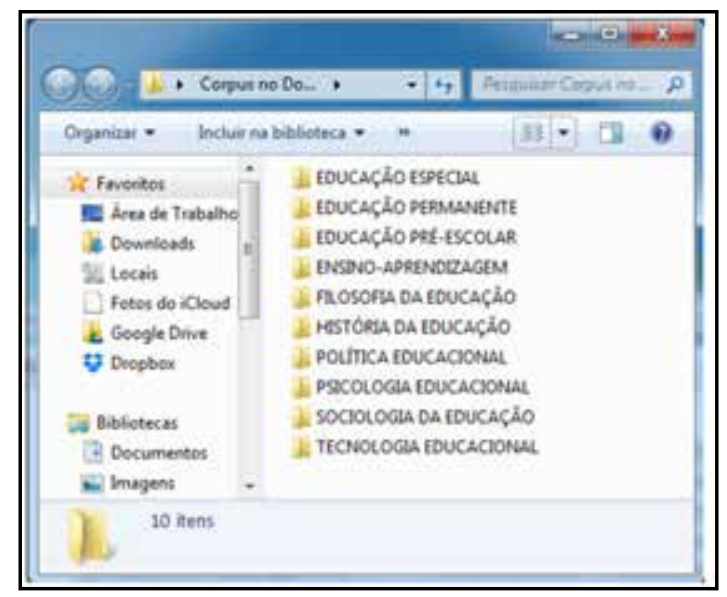

Dentro de cada categoria, o corpus foi divido em cinco pastas, conforme mostra a figura 2. Na primeira pasta, "Anotação", contém as estatísticas e referências externas do texto; na segunda, "Artigos Originais", armazenam-se os artigos originais no formato PDF. Na pasta "palavraschave" são armazenadas aquelas escolhidas por cada autor do artigo, essas palavras podem ser utilizadas no processo de sumarização com a finalidade de melhorar a qualidade do sumário. A pasta "Resumos" traz os resumos manuais de cada artigo, estes são importantes para realizar a avaliação dos sumários automáticos, e, por fim, a pasta "Textos" armazena os corpos dos artigos, que serão submetidos aos sumarizados.

3 Disponível em: https://buscadorcoruja.com/ 
Figura 2. Conteúdo de cada categoria.

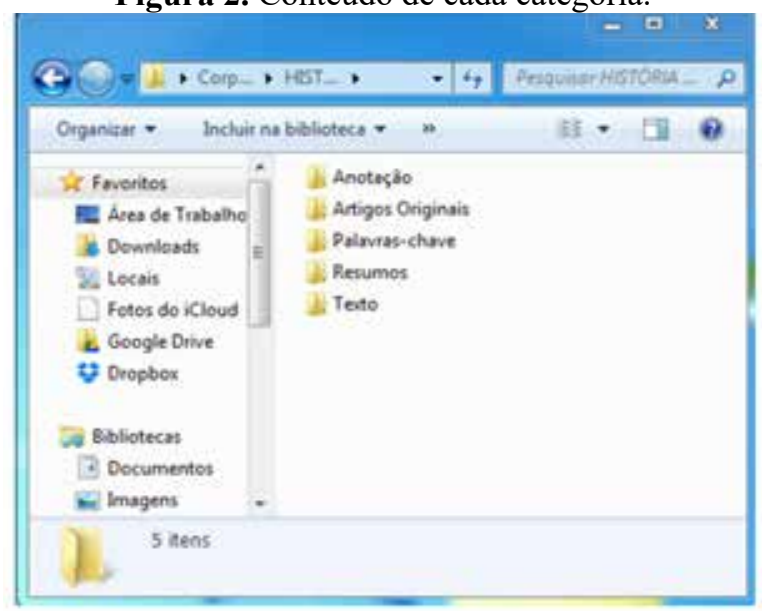

Os arquivos dentro das pastas "Palavras-chave", "Resumos" e "Texto" foram nomeados segundo um padrão no qual o nome do arquivo é formado pelo nome da pasta que ele está inserido e um número de 01 a 50, dessa maneira os arquivos referentes a um mesmo artigo têm o mesmo número. Assim, o artigo armazenado na pasta "Artigos Originais" com o número 02 terá suas respectivas informações nas outras pastas com o nome "chave02", "resumo02" e "texto02". Esse padrão pode ser visualizado na figura 3.

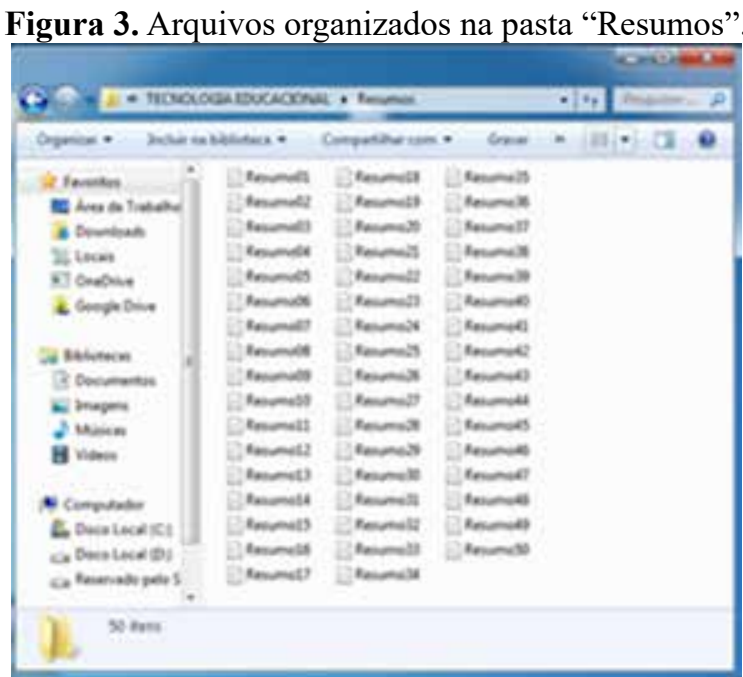


Na figura 4 é apresentado o diagrama do Corpus em Educação. O corpus possui 500 arquivos de texto, sendo 50 textos para cada categoria.

Concluindo, na última fase tem-se a anotação, que se compreende como a retirada dos métodos que, segundo Aloísio e Almeida (2006), são dados estruturados sobre dados, isto é, dados bibliográficos comuns, de catalogação, tais como tamanho do arquivo, tipo da autoria, a tipologia textual e informação sobre a distribuição do corpus, neste caso específico, a retirada dos dados estatísticos do corpus gerado. As referências externas deste corpus se encontram nas pastas "Anotação".

Figura 4. Diagrama do Corpus em Educação
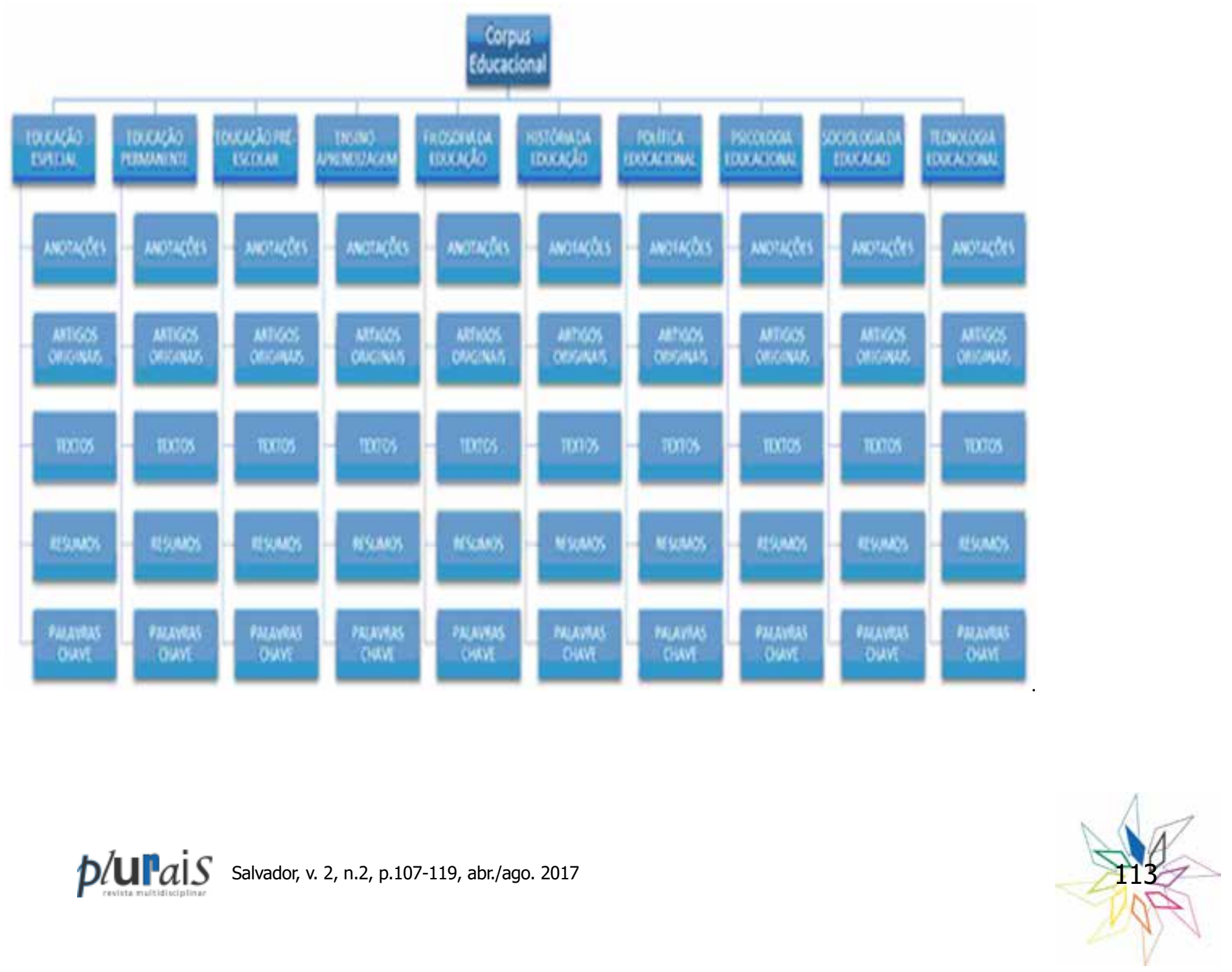


\section{Estatística do corpus}

Esta seção mostra as estatísticas do corpus em educação. Segundo Peixoto e Brito (2015), a LC e a existência de corpora de grande tamanho seriam inconcebíveis sem que houvesse o auxílio de ferramentas computacionais, uma vez que a área trabalha com grandes quantidades de informação textual, impossíveis de se processar manualmente com rapidez, mesmo que por grandes equipes. Portanto, são usados programas de análise lexical para efetuar operações de processamento da linguagem, tais como contagem de palavras, geração de listas de frequência, geração de listas de palavras-chave e exibição de linhas de concordância.

Neste trabalho, as informações estatísticas do corpus foram coletadas através do software FineCount 2.6 free 4 . O corpus é formado por 2.999 .646 palavras no total, cuja distribuição se dá nos 500 artigos selecionados. A tabela 1 sintetiza as estatísticas dos textos separadas nas 10 categorias que compõem o corpus, mostra também o número de palavras por categorias e o número médio de palavras por texto de cada uma destas. De acordo com a tabela, a média de palavras por categoria é 299964,6 e a média por texto é de 5999,29.

Tabela 1. Estatísticas dos textos fonte do corpus.

\begin{tabular}{|c|c|c|c|c|c|c|}
\hline Arquivos & Caracteres & $\begin{array}{c}\text { Caracteres e } \\
\text { espaços }\end{array}$ & Palavras & $\begin{array}{c}\text { Palavras e } \\
\text { numerais }\end{array}$ & $\begin{array}{c}\text { Sentenças } \\
\text { Média de } \\
\text { palavras por } \\
\text { texto }\end{array}$ \\
\hline EDUCAÇÃO ESPECIAL & 1389371 & 1649313 & 274182 & 281052 & 10267 & 5483,64 \\
\hline EDUCAÇÃO PERMANENTE & 1391696 & 1652722 & 281035 & 285382 & 14274 & 5620,7 \\
\hline ENSINO-APRENO PRÉ-ESCOLAR & 1442290 & 1722467 & 281091 & 286480 & 23248 & 5621,82 \\
\hline FILOSOFIA DA EDUCAÇÃO & 1514556 & 1810378 & 313779 & 317365 & 18554 & 6275,58 \\
\hline HISTÓRIA DA EDUCAÇÃO & 1918883 & 2283568 & 392515 & 400849 & 14646 & $\mathbf{7 8 5 0 , 3}$ \\
\hline POLÍTICA EDUCACIONAL & 1846727 & 2188932 & 371540 & 379529 & 12607 & $\mathbf{7 4 3 0 , 8}$ \\
\hline PSICOLOGIA EDUCACIONAL & 1365382 & 1616528 & 266666 & 271889 & 13157 & 5333,32 \\
\hline SOCIOLOGIA DA EDUCAÇÃO & 1643482 & 1952429 & 329689 & 334182 & 17144 & 6593,78 \\
\hline TECNOLOGIA EDUCACIONAL & 1074809 & 1275323 & 213745 & $\mathbf{2 1 8 3 4 5}$ & $\mathbf{7 6 8 8}$ & $\mathbf{4 2 7 4 , 9}$ \\
\hline Total & $\mathbf{1 4 9 6 1 6 9 1}$ & $\mathbf{1 7 7 8 5 8 3 6}$ & $\mathbf{2 9 9 9 6 4 6}$ & $\mathbf{3 0 5 4 8 8 2}$ & $\mathbf{1 4 4 8 8 4}$ & $\mathbf{5 9 9 9 2 , 9 2}$ \\
\hline Desvio Padrão & $\mathbf{2 4 9 0 6 3 , 7 2}$ & $\mathbf{2 9 6 4 0 7 , 3 9}$ & $\mathbf{5 3 0 0 7 , 4 5}$ & $\mathbf{5 4 0 4 2 , 2 4}$ & $\mathbf{4 3 6 1 , 1 3}$ & $\mathbf{1 0 6 0 , 1 4}$ \\
\hline Média Geral & $\mathbf{1 4 9 6 1 6 9 , 1}$ & $\mathbf{1 7 7 8 5 8 3 , 6}$ & $\mathbf{2 9 9 9 6 4 , 6}$ & $\mathbf{3 0 5 4 8 8 , 2}$ & $\mathbf{1 4 4 8 8 , 4}$ & $\mathbf{5 9 9 9 , 2 9}$ \\
\hline
\end{tabular}

4 Disponível em: http://www.tilti.com/software-for-translators/finecount/ 
Na Tabela 2, são mostradas as estatísticas dos sumários manuais dos 10 domínios que formam o corpus, há um total de 168993 palavras e uma média geral de 3379,86 por texto.

Tabela 2. Estatísticas dos Sumários Manuais

\begin{tabular}{|c|c|c|c|c|c|c|}
\hline Arquivos & Caracteres & $\begin{array}{l}\text { Caracteres e } \\
\text { espaços }\end{array}$ & Palavras & $\begin{array}{l}\text { Palavras e } \\
\text { numerais }\end{array}$ & Sentenças & $\begin{array}{l}\text { Média de } \\
\text { palavras por } \\
\text { texto }\end{array}$ \\
\hline EDUCAÇÃO ESPECIAL & 116123 & 135793 & 22633 & 22899 & 723 & 452,66 \\
\hline $\begin{array}{l}\text { EDUCAÇÃO } \\
\text { PERMANENTE }\end{array}$ & 91635 & 107240 & 18425 & 18654 & 628 & 368,5 \\
\hline $\begin{array}{l}\text { EDUCAÇÃO PRÉ- } \\
\text { ESCOLAR }\end{array}$ & 81817 & 96157 & 16054 & 16279 & 760 & 321,08 \\
\hline $\begin{array}{c}\text { ENSINO- } \\
\text { APRENDIZAGEM }\end{array}$ & 88281 & 103381 & 17413 & 17593 & 553 & 348,26 \\
\hline $\begin{array}{l}\text { FILOSOFIA DA } \\
\text { EDUCAÇÃO }\end{array}$ & 77857 & 92091 & 16240 & 16304 & 595 & 324,8 \\
\hline $\begin{array}{l}\text { HISTÓRIA DA } \\
\text { EDUCAÇÃO }\end{array}$ & 71999 & 84727 & 14804 & 14984 & 420 & 296,08 \\
\hline $\begin{array}{c}\text { POLÍTICA } \\
\text { EDUCACIONAL }\end{array}$ & 76407 & 89677 & 15506 & 15694 & 429 & 310,12 \\
\hline $\begin{array}{l}\text { PSICOLOGIA } \\
\text { EDUCACIONAL }\end{array}$ & 81145 & 94957 & 15594 & 15826 & 693 & 311,88 \\
\hline $\begin{array}{l}\text { SOCIOLOGIA DA } \\
\text { EDUCAÇÃO }\end{array}$ & 77136 & 90700 & 15320 & 15368 & 675 & 306,4 \\
\hline $\begin{array}{l}\text { TECNOLOGIA } \\
\text { EDUCACIONAL }\end{array}$ & 88193 & 103060 & 17004 & 17341 & 603 & 340,08 \\
\hline Total & 850593 & 997783 & 168993 & 170942 & 6079 & 3379,86 \\
\hline Desvio Padrão & 12535,78 & 14464,25 & 2289,02 & 2326,87 & 115,01 & 45,78 \\
\hline Média Geral & 81481 & 95557 & 16147 & 16291,5 & 615,5 & 322,94 \\
\hline
\end{tabular}

A Figura 5 mostra a disposição da porcentagem do total de palavras bem como os números dos textos fonte das 10 categorias que constituem o corpus. A variação do tamanho dos textos fonte é pequena, conforme mostrado no gráfico. Esse balanceamento, de acordo com 
Aloísio e Almeida (2006), é um requisito que impacta na validade e confiabilidade do corpus computadorizado. A categoria Tecnologia Educacional possui os textos com a menor quantidade de palavras e numerais, por sua vez, as categorias Política Educacional e História da Educação possuem os maiores textos.

Figura 5. Divisão da porcentagem de palavras e números por categoria dos textos fontes.

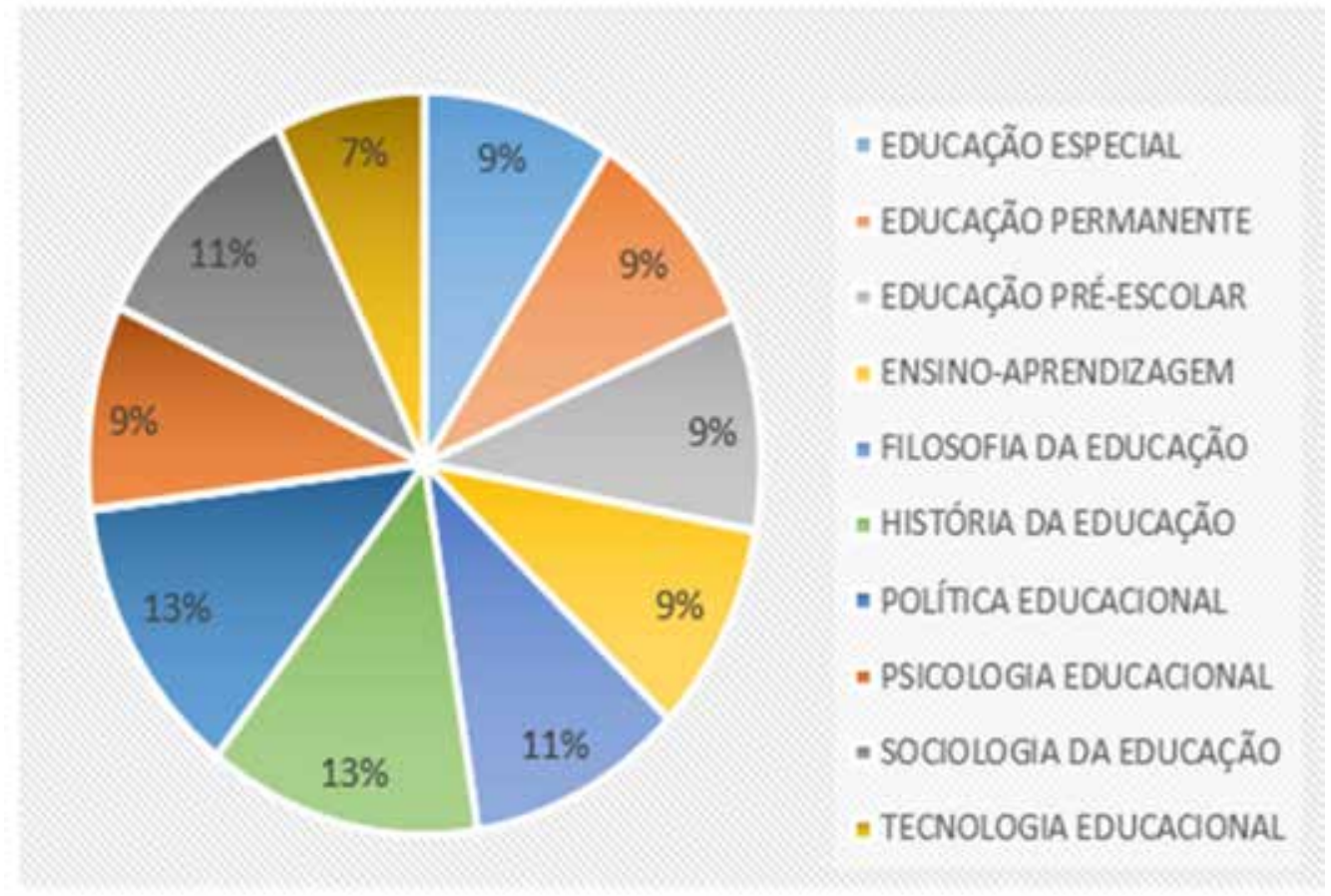

A Figura 6 traz as porcentagens desta distribuição para os sumários manuais. Mostra um balanceamento entre a quantidade de palavras e números das 10 categorias do corpus. 
Figura 6. Divisão da porcentagem de palavras e números por categoria dos sumários Manuais

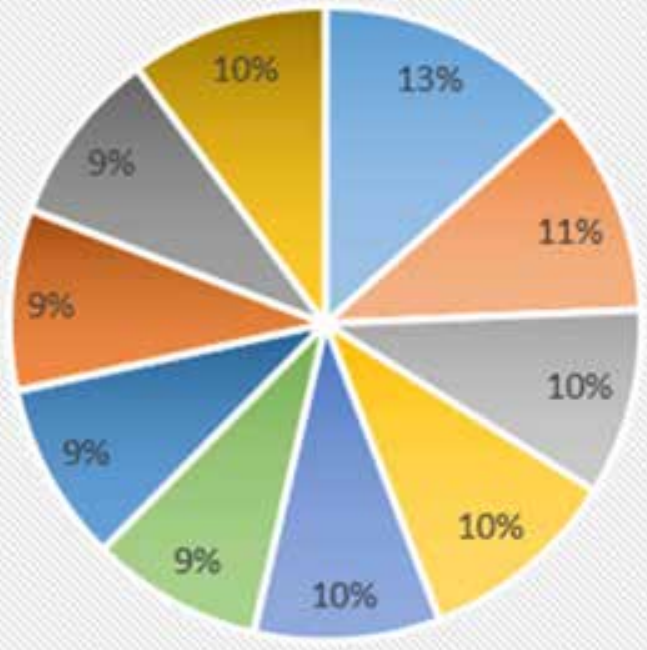

- EDUCAÇĀO ESPECLAL

- EDUCAÇÃO PERMANENTE

- EDUCAÇÃO PRÉ-ESCOLAR

- ENSINO-APRENDIZAGEM

- FILOSOFIA DA EDUCAÇ̃̃o

- HISTÓRIA DA EDUCAÇÃo

- POLITICA EDUCACIONAL

- PSICOLOGIA EDUCACIONAL

- SOCIOLOGIA DA EDUCAÇ̃̃o

- TECNOLOGIA EDUCACIONAL

\section{Conclusão}

Este artigo descreve os procedimentos para a construção e as estatísticas de um corpus que foi construído a partir de artigos científicos na língua portuguesa e de domínio educacional. Espera-se poder contribuir com a comunidade científica ao disponibilizar este corpus para simulação.

O corpus construído neste trabalho faz parte dos estudos desenvolvidos no grupo de pesquisa Mineração de Textos e Processamento de Linguagem Natural e Aprendizado de Máquina (MTPLNAM), que também já produziu outros corpora, conforme os trabalhos de Oliveira e Guelpeli (2014), Fernandes e Guelpeli (2014) e Guelpeli e Fernandes (2016).

O grupo MTPLNAM tem como objetivo pesquisar, gerar conhecimentos e desenvolver aplicações sobre mineração de texto (MT), processamento de linguagem natural (PLN) e aprendizagem de máquina (AM), sua página pode ser acessada em http://www.mtplnam.com.br, 
onde estão outros corpora produzidos pelo grupo e disponibilizados para a comunidade científica para testes e trabalhos.

A necessidade deste corpus é viabilizar os estudos na área de sumarização automática e possibilitar que sumarizadores possam ser avaliados por ferramentas especializadas, como o ROUGE (Recall-Oriented Understudy for Gisting Evaluation) (LIN e HOVY, 2003), além de dar suporte a sumarizadores que utilizam palavras-chave para personalizar seus sumários, como é feito no sumarizador PragmaSUM (ROCHA, 2014), que também é um projeto do grupo MTPLNAM.

Assim, este corpus tem a finalidade de contribuir com a continuidade dos estudos da eficiência de sumarizadores, com possibilidade de utilização das palavras-chave no processo de sumarização dos textos, além de viabilizar estudos da influência destas palavras nos conteúdos dos textos em questão.

\section{REFERÊNCIAS}

ALUÍSIO, S.M.; ALMEIDA, G.M.B. O que é e como se constrói um corpus? Lições aprendidas na compilação de vários corpora para pesquisa linguística. Calidoscópio, (UNISINOS). vol. 4, n. 3, p. 155-177, set/dez 2006.

FERNANDES, H M; GUELPELI, M. V. C. Creación de corpus en lengua española para su utilización en testes acerca de Sumarización Automática. In: 6th International Conference on Corpus Linguistics-CILC 2014, 2014, Las Palmas de Gran Canaria. 6th International Conference on Corpus Linguistics-CILC 2014, 2014.

GUELPELI, M. V. C.; FERNANDES, H. M. Input a Word, Analyze the World: Selected Approaches to Corpus Linguistics...ISBN-13: 978-1-4438-8513-3 e ISBN-10: 1-4438-8513$41^{\text {a }}$. ed. United Kingdom: Cambridge Scholars Publishing, 2016. v. I. 521p

GUELPELI, M.V.C. (2012) "Cassiopeia: Um Modelo de Agrupamento de Textos Baseado em Sumarização". http://nlx.di.fc.ul.pt/ guelpeli/Arquivos/Tese.pdf.

HUMBLÉ, Philippe. Dictionaries and Language Leaners. Frankfurt am Main, 2001. 
KUCERA, H and FRANCIS W. N. Brown University Standard Corpus of Present-Day American English (or just Brown Corpus) as a general corpus (text collection) in the field of corpus linguistics. It contains 500 samples of English-language text, totalling roughly one million words, compiled from works published in the United States in 1961.

LIN, C-Y. and HOVY, E.H. Automatic Evaluation of Summaries Using N-gram Cooccurrence Statistics. In the Proceedings of Language Technology Conference - HLT. Edmonton, Canadá, 2003.

MARTINS, M S. CASELI, H M. NUNES, M G V N. A construção de um corpus de textos paralelos inglês-português. Série de Relatórios do Núcleo Interinstitucional de Linguística Computacional NILC - ICMC-USP, Caixa Postal 668, 13560-970 São Carlos, SP, Brasil, Set. 2001.

OLIVEIRA, R.R.; GUELPELI, M.V.C. Building a Corpus in Italian Written Language. In:6th International Conference on Corpus Linguistics (CILC2014). Las Palmas de Gran Canaria, Espanha, 2014. No prelo.

PEIXOTO, L. M.; BRITO, L. F. A. Procedimentos para compilação de um corpus composto por legendas e construção de uma ferramenta de corpus on-line: o Corpus of English Language Videos. DOMÍNIOS DE LINGU@GEM. <http://www.seer.ufu.br/index.php/dominiosdelinguagem> - v. 9, n. 3 (jul/set. 2015) - ISSN 1980-5799.

REBECHI, Rozane R.; ANDREETTO, Marlene D. As retraduções de Trauerund Melancholie para o português: o léxico freudiano sob o olhar da Linguística de Corpus. Pandaemoniumger., São Paulo, v. 18, n. 26, p. 126-157, Dec. 2015. Available from $<$ http://dx.doi. org/10.1590/1982-88371826126157>. access on 30 May 2016.

ROCHA, V. J. C., PragmaSUM: Um Sumarizador Automático De Textos Baseado Em Perfil De Usuário. Trabalho de Conclusão de Curso de Sistemas de Informação da Universidade Federal dos Vales do Jequitinhonha e Mucuri. Diamantina- MG, Brasil, 2014.

SARDINHA, T. B. (2000) "Linguística de Corpus: Histórico e Problemática". DELTA, São Paulo, v. 16, n. 2, p 323-367, 2000a <http:/www.scielo.br/scielo.php?script=sci_arttext\&pi$\underline{\mathrm{d}=\mathrm{S} 0102-44502000000200005>}$

ZAVAGLIA, C. FERRARESI, M. L. Construção de um corpus paralelo e alinhado português italiano-português para o domínio literário. Estudos Linguísticos XXXV, p. 502-511, 2006. 